\title{
Examining the impact of age and multitasking on motorcycle conspicuity
}

\author{
Jonathan L. Ledbetter, Michael W. Boyce, Drea K. Fekety, Ben Sawyer, and Janan A. Smither Ph.D. \\ Applied Experimental and Human Factors Psychology, University of Central Florida, 4000 Central Florida Blvd., \\ Orlando, FL, U.S.A.
}

\begin{abstract}
This poster presents a study to assess one's ability to detect motorcycles under different conditions of conspicuity while performing a secondary visual load task. Previous research in which participants were required to detect motorcycles revealed differences in age (young adults/older adult) as well as differences associated with motorcycle conspicuity conditions. Past research has specifically found motorcycles with headlights $\mathrm{ON}$ and modulating headlights (flashing) to be more conspicuous than motorcycles with headlights OFF within traffic conditions [1]. The present study seeks to provide more information on the effects of multitasking on motorcycle conspicuity and safety. The current study seeks to determine the degree to which multitasking limits the conspicuity of a motorcycle within traffic. We expect our results will indicate main effects for distraction task, age, gender, motorcycle lighting conditions, and vehicular DRLs on one's ability to effectively detect a motorcycle. The results have implications for motorcycle safety in general and through this research, a better understanding of motorcycle conspicuity can be established so as to minimize the risk involved with motorcycle operation.
\end{abstract}

Keywords: Conspicuity, attention, motorcycle, driving performance, multitasking

\section{Introduction}

Previous research in which participants were required to detect motorcycles revealed differences in age (young adults/older adults) as well as differences associated with motorcycle conspicuity elements. Specifically, motorcycles with headlights $\mathrm{ON}$ or headlights modulating (flashing) are more conspicuous than motorcycles with headlights OFF within traffic conditions [1].

This research seeks to provide more detailed information on the effects of multitasking on motorcycle conspicuity and safety. This research also seeks to determine the degree to which multitasking limits the effectiveness of motorcycle conspicuity within traffic. The results have implications for motorcycle safety in general and through this research, a better understanding of motorcycle conspicuity can be established so as to minimize the risk involved with motorcycle operation.

\section{Methods}

\subsection{Participants}

Approximately 100 male and female participants will take part in this experiment. This experiment will include three groups separated by age, one consisting of younger adults (18 -30 yrs), one consisting of middle aged adults $(40-55 \mathrm{yrs})$, and one consisting of older adults ( $65 \mathrm{yrs}$ and above).

Participants in the young adult group will be recruited via an electronic listing within the UCF Psychology recruitment service, SONA Systems. Participants in the middle and older adult groups will be recruited via flyers that will be posted at various locations within the community immediately surrounding UCF. Participants in the older adult group will also be recruited via flyers handed out during monthly LIFE at UCF meetings and at various senior centers in the Orlando area.

All participants will be screened for visual acuity, using a STEREO OPTEC 2000 vision testing system, where only participants with 20/40 (corrected) for visual acuity will be included.

Participants will be screened for motorcycle experience. Only participants without prior first hand motorcycle experience will be included in this study. "Motorcycle experience" in this study is defined as current/past operation of a motorcycle or moped and/or close relations with an individual with current/past experience operation of a motorcycle or moped. 
Participants in the study will be granted "Experimental Course Credit", if applicable, for participating in this experiment.

\subsection{Distraction Task}

The distraction task used for this study was developed out of the research of Kennedy, May, Dunlap, Jones, and Smith [2]. This distraction task consists of a four channel stimulus where four boxes are displayed on a computer screen. Each box is presented individually and the participant responds as quickly as possible to the presentation of the box.

In the present study, the original distraction task was modified to encompass three channels (three boxes) instead of four. Each box is presented individually at irregular intervals. The participant is asked to count each presentation of the stimulus and respond to every fourth presentation on each channel. The program is designed to measure accuracy and response latency.

\subsection{Procedure}

Participants will be run through practice sessions in the secondary distracter task as well as the primary hazard detection scenario, to familiarize them with the controls for the testing environment.
Once these practice sessions are completed, they will begin the first of two hazard-detection sessions involving approximately 40 video clips of road situations. A second session of the same type will follow immediately afterwards. The reason for having two sessions is so that participants can have exposure to the hazard-detection environment with and without the secondary distracter task.

\section{Summary}

This research is currently underway. The purpose of this study is to assess one's ability to detect motorcycles under different conditions of conspicuity while performing a secondary visual load task. Measurements taken during this study will include: reaction time, distance to detection, hits/misses as well as false alarms.

\section{References}

[1] J. A. Smither and L. I. Torrez, Motorcycle conspicuity: Effects of age and daytime running lights, Human Factors 52 (2010), 355-369.

[2] R. S. Kennedy, J. G. May, W. P. Dunlap, M. B. Jones and M. B. Smith, An automated task load indexing system, Technical report N62269-90-C-0279, Essex Corporation, 1991. 